\title{
Crustáceos decápodos de las cuencas Copalita, Zimatán y Coyula, en Oaxaca, México
}

\section{Decapod crustaceans from Copalita, Zimatán and Coyula basins, in Oaxaca, Mexico}

\author{
José Luis Villalobos-Hiriart ${ }^{1 *}$, Fernando Álvarez ${ }^{1}$ Carmen Hernández ${ }^{1}$ Guadalupe de la Lanza-Espino² e \\ Ignacio Daniel González-Mora ${ }^{3}$
}

${ }^{1}$ Colección Nacional de Crustáceos, Instituto de Biología, Universidad Nacional Autónoma de México. Apartado postal 70-153, 04510 México, D.F., México.

${ }^{2}$ Laboratorio de Hidrobiología, Instituto de Biología, Universidad Nacional Autónoma de México. Apartado postal 70-153, 04510 México, D.F., México.

${ }^{3}$ Programa Manejo de Cuencas Copalita-Zimatán-Huatulco, World Wildlife Fund-México. Calle Circuito Acueducto 320 , Fraccionamiento Rincón del Acueducto 68040 Oaxaca, Oaxaca, México.

*Correspondencia: hiriart@servidor.unam.mx

\begin{abstract}
Resumen. Se estudiaron 13 localidades de las cuencas Copalita, Zimatán y Coyula, Oaxaca. En 4 muestreos se capturaron más de 3200 crustáceos decápodos representando 3 familias, 4 géneros y 13 especies, y 3 posibles nuevas especies de los géneros Atya y Pseudothelphusa. Las especies se analizaron de acuerdo con su abundancia, frecuencia de aparición y distribución en las cuencas, y se comparó la composición de especies y distribución altitudinal de las localidades. La familia mejor representada fue Palaemonidae con 1 género y 8 especies, le siguieron Atyidae con 2 géneros y 3 especies y Pseudothelphusidae con 1 género y 3 especies. Potimirim glabra y Atya sp. 1 fueron las especies más abundantes; $A$. margaritacea y P. glabra las más frecuentes, pues se detectaron en 10 de 13 estaciones; Macrobrachium michoacanus se detectó en 9, y M. americanum y M. occidentale en 8 estaciones. Los juveniles de langostino tuvieron una alta frecuencia en 9 de las localidades. La riqueza y el índice de diversidad de especies se incrementaron hacia la porción baja de los ríos; en la porción alta, los valores fueron bajos y en la media de moderados a altos. Las localidades de muestreo se asociaron en 3 grupos de acuerdo con su altitud.
\end{abstract}

Palabras clave: Crustacea, Decapoda, dulceacuícola, Oaxaca, México.

\begin{abstract}
Thirteen localities were studied in the Copalita, Zimatán, and Coyula basins, Oaxaca. More than 3200 decapod crustaceans were captured representing 3 families, 4 genera, and 13 species, including 3 possible new species of Atya and Pseudothelphusa. Abundance, frequency and within basin distribution of species were analyzed. Collecting localities were compared considering their altitude and species composition. The family Palaemonidae had the most species grouped in 1 genus with 8 species, the family Atyidae had 2 genera and 3 species and the family Pseudothelphusidae contributed with 1 genus and 3 species. The most abundant species were Potimirim glabra and Atya sp. 1. Atya margaritacea and Potimirim glabra were the most frequent species occurring in 10 of the 13 sites, Macrobrachium michoacanus occurred in 9, and M. americanum and M. occidentale in 8. The juveniles of Macrobrachium occurred in 9 sites. Species richness and the diversity index tended to increase towards the low portions of the rivers; in the upper reaches these values were low and intermediate to high in the middle portions of the basins. Sampling sites could be classified in 3 groups according to their altitude.
\end{abstract}

Key words: Crustacea, Decapoda, freshwater, Oaxaca, Mexico.

\section{Introducción}

En el ambiente dulceacuícola de México, los crustáceos integran un grupo heterogéneo en el cual es posible distinguir formas de varios componentes zoogeográficos;

Recibido: 02 febrero 2010; aceptado: 13 mayo 2010 los más característicos son los relacionados con las regiones neártica y neotropical de América (Villalobos-Figueroa et al., 1982; Villalobos-Hiriart et al., 1993). Para el orden Decapoda se han registrado cerca de 160 especies que se distribuyen hasta altitudes mayores de los 3000 metros.

A pesar de la diversidad de crustáceos decápodos que se puede encontrar en las aguas continentales de México, 
el conocimiento que se tiene de ellos es moderado y está lejos de ser el más completo, lo que aunado a la acelerada pérdida de dichos ambientes, debida a que el agua dulce es uno de los recursos no renovables estratégicos para el país, lleva a suponer que la mayoría de las especies se encuentran en riesgo o están amenazadas, en particular las endémicas que constituyen más del $80 \%$ para México (VillalobosHiriart et al., 1993; Villalobos y Álvarez, 2008).

La información sobre los crustáceos decápodos dulceacuícolas de Oaxaca se encuentra dispersa en diversas publicaciones, sin que hasta el momento se tenga un estudio que integre la riqueza de especies y su distribución a través del estado. Resaltan los artículos donde se describen especies nuevas oaxaqueñas registradas en localidades colindantes con los estados de Veracruz y Chiapas, así como en cuerpos de agua dulce de la vertiente occidental del estado (Rathbun, 1898, 1933; Villalobos, 1948, 1967; Rodríguez y Smalley, 1969; Hobbs, 1973a, b; Holthuis, 1977; Álvarez y Villalobos, 1991; Villalobos y Álvarez, 2003; Mejía-Ortiz et al., 2003; Mejía-Ortiz et al., 2003). También se cuenta con estudios monográficos sobre algún género o familia de los decápodos de agua dulce de México o de América, donde se mencionan localidades de Oaxaca (Holthuis, 1952; Villalobos, 1954, 1955, 1960, 1969, 1982; Hobbs y Hart, 1982; Rodríguez, 1982, 1992; Hobbs, 1989; Rojas-Paredes, 1998; Mejía-Ortíz, 2005; Villalobos-Hiriart, 2005; Villalobos y Álvarez, 2008).

Hasta el momento no existen estudios sobre Oaxaca para los que se hayan realizado recolecciones periódicas en una cuenca hidrológica y que abarquen todas las temporadas de un ciclo anual. A partir del proyecto "Propuesta de indicadores de salud de la cuenca para el monitoreo hidrológico, Copalita-Zimatán-Huatulco, Oaxaca" financiado por World Wildlife Fund y la Fundación Gonzálo Río Arronte, I.A.P., se presenta este estudio como línea de base para evaluar el impacto de futuros desarrollos en la región.

Área de estudio. El complejo hidrológico Copalita-ZimatánCoyula se localiza en la vertiente costera occidental de la sierra Madre del Sur en el estado de Oaxaca, cubre una superficie total de 268023 ha, y se extiende de los $15^{\circ} 40^{\prime}$ a $\operatorname{los} 16^{\circ} 00^{\prime} \mathrm{N}$ y de los $96^{\circ} 00^{\prime}$ a los $96^{\circ} 30^{\prime} \mathrm{O}$ (Fig. 1). Fisiográficamente, la región pertenece a la provincia Sierra Madre del Sur, el sustrato presenta una gran variedad de rocas, tanto por su origen como por su edad, entre las que destacan complejos metamórficos de edad paleozoica y gneises de edad jurásica. Está comprendida dentro de los terrenos tectonoestratigráficos Oaxaca y Xolapa; el primero, constituido por el basamento más antiguo del sur de México, se denomina Complejo Oaxaqueño y está representado por una variedad de rocas metamórficas (INEGI, 1996).
De acuerdo con la clasificación de la Comisión Nacional del Agua, el sistema fluvial Copalita-Zimatán-Coyula pertenece a la Región Hidrológica 21: Costa de Oaxaca (www.inegi.org.mx). Las cuencas se caracterizan por llevar agua todo el año, desembocan al océano Pacífico y en época de lluvias se convierten en corrientes torrenciales, ya que captan los escurrimientos provenientes de la sierra Madre del Sur. La cuenca del río Copalita abarca 152945 ha y la constituyen las subcuencas Copalita, Yuviaga, Pluma Hidalgo y río Hondo. Nace cerca del parteaguas de la sierra Madre del Sur, en San Jerónimo Ozolotepec al norte de Pluma Hidalgo, toma una dirección SE y desagua al Pacífico por la barra de Copalita, al sur de Pochutla (Tamayo, 1949). La cuenca del río Zimatán abarca 47270 ha y tiene cerca de $60 \mathrm{~km}$ de longitud. El río nace al sur de San Pablo Totiltepec, municipio de San Carlos Yautepec, colinda al norte con La Merced del Potrero, al sur con el océano Pacífico, al este con San Isidro Chacalapa y al oeste con San Miguel del Puerto. A lo largo del río se encuentran las comunidades de La Merced del Potrero, Santa María Xadani, Santa María Petatengo, San Lorenzo y Zimatán; la altitud va desde los 0 a los 1400 m; en la parte alta se localizan zonas cafetaleras. En esta cuenca se ubican los municipios de San Miguel del Puerto, San Carlos Yautepec, Santiago Astata y San Pedro Huamelula, pertenecientes a los distritos de Pochutla y Tehuantepec de las regiones Costa e Istmo, respectivamente (GonzálezMora et al., 2009). La cuenca del río Coyula o Huatulco es la más corta y la más contaminada de las 3 ; está formada por los ríos Magdalena y Cruz (Huatulco), tiene una superficie de 41323 ha y se compone de 10 microcuencas que corren de manera perpendicular a la línea de costa; en su trayecto atraviesa el poblado de Santa María Huatulco, del que recoge aguas residuales. Aunque existen plantas de tratamiento (río Laje y en los parajes de El Azulillo y El Morro), los pobladores de los Bajos de Coyula y Puente Coyula han manifestado afectaciones por aguas negras contaminadas (González-Mora et al., 2009).

Debido al amplio intervalo altitudinal del área de estudio (0-1 $500 \mathrm{~m})$ se registraron diversos tipos de vegetación, que van desde las selvas bajas espinosas caducifolias hasta bosques mesófilos de montaña y bosques templados de pino-encino, pasando por grandes superficies de selvas secas, subhúmedas y húmedas (Salas-Morales et al., 2003).

\section{Materiales y métodos}

Localidades. El área de estudio se dividió en 13 localidades con el objetivo de tener una representación en 3 niveles de altitud de las cuencas Copalita, Zimatán y Coyula (Fig. 


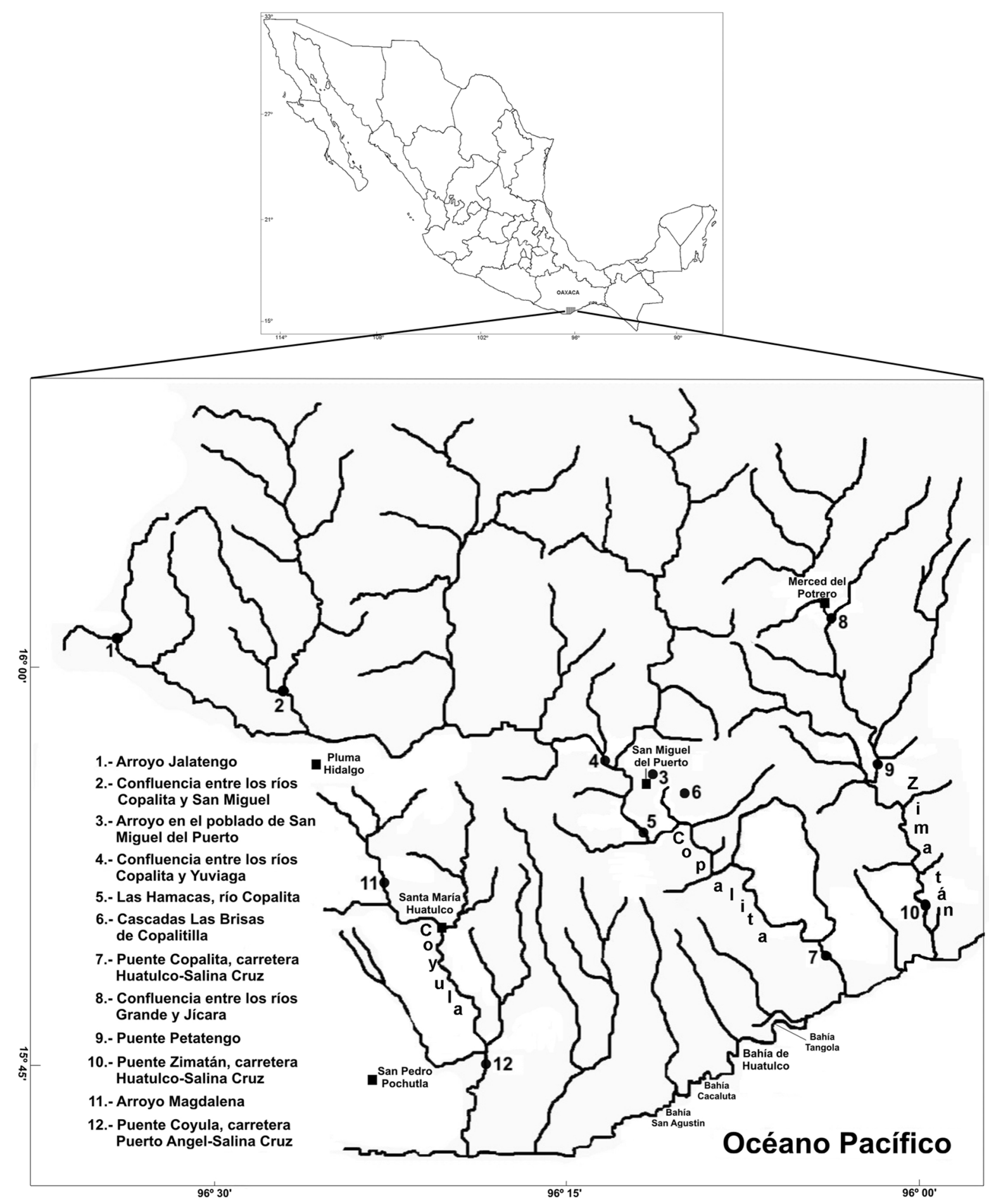

Figura 1. Área de estudio y ubicación de las localidades muestreadas en los ríos Coyula, Copalita y Zimatán. 
1). En lo que se denominó "cuenca alta" en el río Copalita se incluyeron las estaciones: Jalatengo 1 y 2 (1 420 m), la confluencia de los ríos San Miguel y Copalita (885 m) y el arroyo ubicado en San Miguel del Puerto (345 m). En la "cuenca media" del río Copalita se establecieron las localidades de Las Brisas de Copalitilla (250 m), Las Hamacas $(155 \mathrm{~m})$ y la confluencia de los ríos Copalita y Yuviaga (140 m), incluyendo el manantial Piedras Negras (350 m); en el río Zimatán se incluyeron la confluencia de los ríos Grande y Jícara (461 m) así como el Puente Petatengo (229 m), y en el río Coyula, el arroyo Magdalena (324 m). En la "cuenca baja" se tiene para el río Copalita la localidad del puente de la carretera Huatulco-Salina Cruz (29 m); para el río Zimatán, puente de la carretera Huatulco-Salina Cruz (14 m), y para el río Coyula, puente de la carretera Puerto Angel-Salina Cruz (24 m).

Trabajo de campo. Los muestreos se efectuaron en enero, abril, junio y octubre de 2007; en diferentes ambientes que presentan los ríos: rápidos, remansos, orillas con vegetación ribereña, rocas con fanerógamas acuáticas y zonas de inundación adyacentes a los márgenes de los ríos. La captura de los ejemplares se realizó de manera manual, o con la ayuda de una red de cuchara, o con un chinchorro pequeño $(1.5 \times 3 \mathrm{~m})$ con una abertura de malla de 0.5 $\mathrm{cm}$. También se examinaron algunas nazas o trampas "chacaleras" usadas por los lugareños. En cada una de las localidades de muestreo se registraron los parámetros fisicoquímicos básicos (tem-peratura, oxígeno disuelto, salinidad, etc.), así como su georreferenciación y altitud.

Trabajo de laboratorio. La revisión de los ejemplares capturados incluyó su identificación taxonómica en los niveles de género o especie, para lo cual se usaron los trabajos de Villalobos (1960) y Hobbs y Hart (1982) para los Atyidae; Holthuis (1952), Nates-Rodríguez y Villalobos-Hiriart (1990) y Hernández et al. (2007) para los Palaemonidae, así como Rodríguez (1982) y Villalobos (2005) para los Pseudothelphusidae. Una vez reconocidos los taxa se procedió al conteo y sexado de los ejemplares, para incluirlos posteriormente en el catálogo de acceso de la Colección Nacional de Crustáceos (CNCR) del Instituto de Biología, Universidad Nacional Autónoma de México. Se registraron algunos datos poblacionales de cada una de las localidades muestreadas: número de ejemplares, de machos, hembras, hembras ovígeras y juveniles.

El análisis ecológico de la comunidad consistió en determinar la riqueza de especies para cada cuenca. Se calcularon diversidad $\left(\mathrm{H}^{\prime}\right)$, diversidad máxima ( $\left.\mathrm{H}^{\prime} \mathrm{max}\right)$, equidad $\left(\mathrm{J}^{\prime}\right)$ y dominancia mediante los índices de Shannon y Wiener, basados en el criterio de Gray (1981) y se obtuvieron los patrones de asociación entre localidades y distribución de las especies de acuerdo con la altitud, mediante la técnica de agrupamiento por distancias euclidianas en el paquete estadístico STATISTICS II® para Windows ${ }^{\circledR}$.

\section{Resultados}

Identidad de las especies. Se recolectó un total de 3287 decápodos, pertenecientes a 3 familias, 4 géneros y 13 especies. Además de identificar especies ya conocidas, se encontraron ejemplares de los géneros Atya y Pseudothelphusa que pueden representar nuevas especies. Laforma reconocida como Atya sp.1 presenta características que la acercan a Atya ortmannioides Villalobos, 1956, que se distribuye en la vertiente occidental de los estados de Guerrero, Michoacán, Colima y Nayarit, con un registro en La Paz, Baja California Sur (Hobbs y Hart, 1982; Villalobos-Figueroa, 1982). Los ejemplares capturados en este estudio presentan los pereiópodos del tercero y del cuarto par más robustos y con una ornamentación muy evidente en la superficie. De acuerdo con la descripción de $A$. ortmannioides deben ser esbeltos, con la superficie lisa o con gránulos bajos (Villalobos, 1956; Hobbs y Hart, 1982).

Entre los cangrejos del género Pseudothelphusa Saussure, 1858, se identificaron 3 formas diferentes: Pseudothelphusa sp. 1, cercana a P. sulcifrons Rathbun, 1898, citada para Villa Hidalgo Yalag, municipio de Villa Hidalgo, Oaxaca; presenta diferencias en la ornamentación del gonópodo del macho en cuanto al número, posición, forma y tamaño de los dientes en el margen lateral del proceso mesial. Pseudothelphusa sp. 2, cercana a P. galloi Álvarez y Villalobos, 1990, citada para el municipio de Ayutla de los Libres (Álvarez y Villalobos, 1990) y para el Parque El Veladero en la bahía de Acapulco, Guerrero (Villalobos-Hiriart, 2005); presenta diferencias en la ornamentación del gonópodo del macho en cuanto a la forma y tamaño de la proyección caudomarginal y al número de dientes en el margen lateral de la misma proyección. Pseudothelphusa sp. 3, cercana a $P$. guerreroensis Rathbun, 1933, citada para Malinaltepec, municipio de Tlapa de Comonfort y para Copanatoyac, municipio de Copanatoyac, ambas localidades en el estado de Guerrero, con un registro adicional en el municipio de Villa Sola de Vega, Oaxaca (CNCR, 1780). En los 3 casos la revisión taxonómica está fuera del marco del presente artículo y será motivo de otras publicaciones.

Por lo menos 6 especies de Macrobrachium con rostro corto se distribuyen en la vertiente occidental de México (la única de rostro largo es $M$. tenellum [Smith, 1871] y ese rasgo ayuda a diferenciarla fácilmente del resto). Los organismos reconocidos como Macrobrachium sp. corresponden a juveniles de este género que no pudieron 


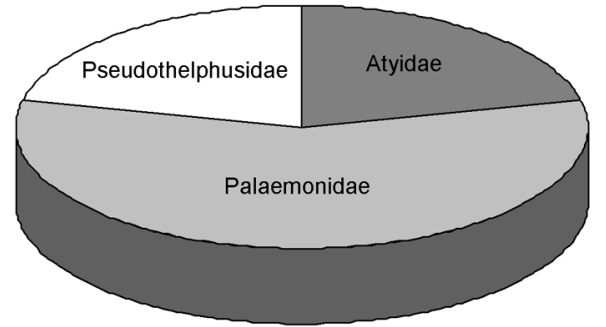

A

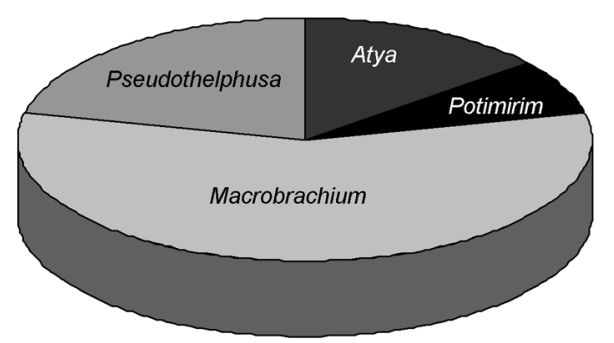

B

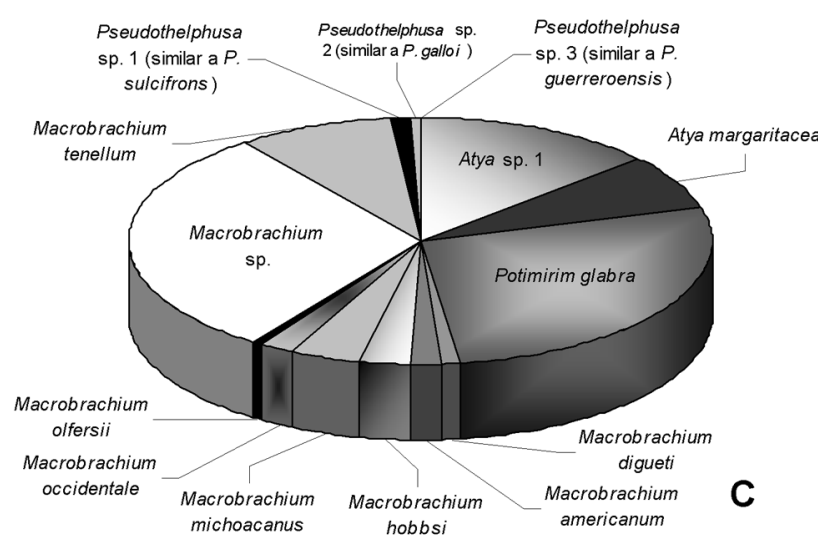

Figura 2. Representación de la proporción de los grupos de crustáceos decápodos registrados: $\mathrm{A}$, número de especies por familia; $\mathrm{B}$, número de especies por género; $\mathrm{C}$, número de organismos por especie.

identificarse a nivel específico, por ello, los datos de abundancia de juveniles son difíciles de interpretar pues no se puede saber cuáles especies están ahí representadas. Riqueza, abundancia y frecuencia de aparición por familia. La familia mejor representada por el número de especies fue Palaemonidae con un género y 7 especies, le siguieron Atyidae con 2 géneros y 3 especies y Pseudothelphusidae con un género y 3 especies (Fig. 2A). En el nivel de género, Macrobrachium presentó 7 especies, seguida por Pseudothelphusa con 3, Atya con 2, y Potimirim con
1 (Fig. 2B). En lo que se refiere a las especies, la más abundante fue Potimirim glabra (Kingsley, 1878) con 918 organismos, seguida por Atya sp. 1 con 449, M. tenellum con 290, y A. margaritacea A. Milne-Edwards, 1864, con 247. La especie con menos organismos capturados fue Pseudothelphusa sp. 3 (similar a P. guerreroensis) con 3 (Fig. 2C). En cuanto a la frecuencia de aparición, las especies de Atya fueron las más constantes, aparecieron en 11 de las 13 localidades. Le siguieron las de los géneros Potimirim, que aparecieron en 10, Macrobrachium en 9, y Pseudothelphusa en 8 localidades (Fig. 3A). Por especie, Atya margaritacea y Potimirim glabra fueron las más frecuentes, apareciendo en 10 de las 13 estaciones; le siguieron M. michoacanus Nates-Rodríguez y VillalobosHiriart, 1990, en 9 estaciones, y M. americanum Bate, 1868, y M. occidentale Holthuis, 1950, aparecieron en 8 estaciones. La forma más rara fue Pseudothelphusa sp. 3; sólo se obtuvo en la confluencia de los ríos San Miguel y Copalita. Los juveniles de langostino (Macrobrachium sp.) aparecieron con gran frecuencia en los puntos de muestreo, se detectaron en 9 de los 13 sitios (Fig. 3B).

Distribución de los crustáceos en las cuencas estudiadas. La presencia de las especies reconocidas en las cuencas de los ríos Copalita, Zimatán y Coyula fue heterogénea, debido principalmente a la diferencia de altitud de las localidades. Hacia la porción baja de los ríos la riqueza de especies tiende en general a incrementarse; en la porción alta fue escasa, y de escasa a moderada en la media (Fig. 4).

En la porción alta del río Copalita el número de especies fue bajo, básicamente representado por cangrejos del género Pseudothelphusa. En Jalatengo se capturaron 4 ejemplares de Pseudothelphusa sp.1;, en la confluencia de los ríos San Miguel y Copalita, 3 de Pseudothelphusa sp. 3, y 1 de Atya margaritacea, camarón de río de amplia distribución a lo largo del Copalita, que se encuentra desde esa altitud $(885 \mathrm{~m})$ hasta las localidades de la cuenca baja. En el arroyo del poblado de San Miguel del Puerto se obtuvieron cangrejos Pseudothelphusa sp. 1 y Potimirim glabra.

En la porción media del río Copalita, en Cascadas Las Brisas de Copalitilla, se obtuvieron 9 especies. Destacó P. glabra por el número de ejemplares (65), con predominancia de hembras (1 $\hat{\gamma}$ vs 64 우). Los juveniles de langostino también están presentes en esta localidad, lo cual sugiere que alguna de las especies de Macrobrachium está completando su ciclo biológico a esa altitud $(250 \mathrm{~m})$ y a una distancia considerable $(\sim 50 \mathrm{~km})$ de los ambientes salobres que requieren para su desarrollo. En Las Hamacas se registraron 8 especies; por el número de ejemplares sobresalen M. michoacanus (26), M. occidentale (19), Atya sp.1 (18) y P. glabra (11). Particularmente en 


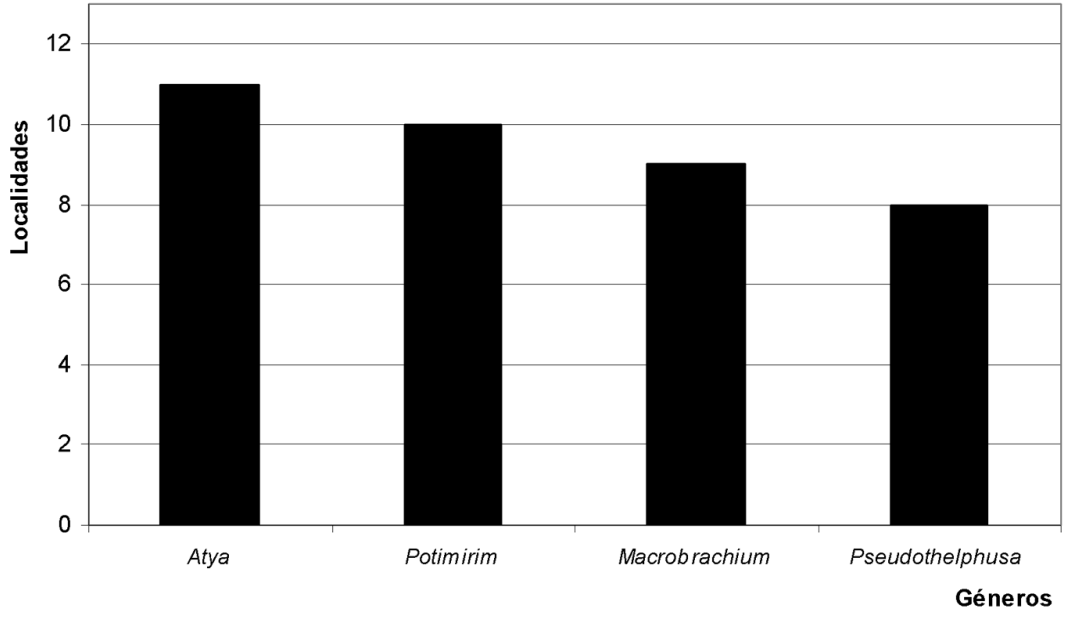

A

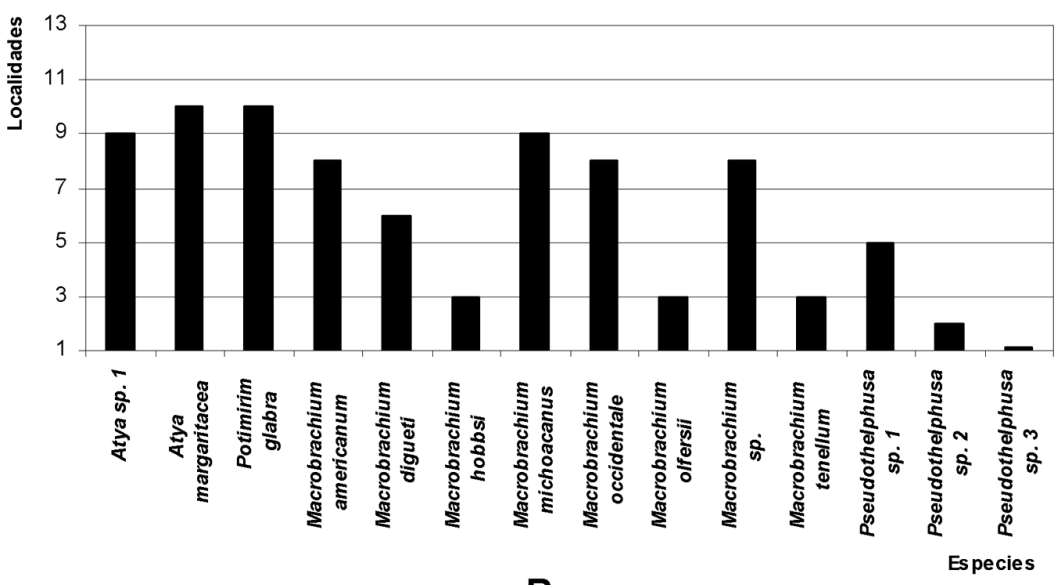

B

Figura 3. Frecuencia de aparición de los grupos de crustáceos decápodos registrados: A, número de localidades en las que se encontró cada género; B, número de localidades en las que se encontró cada especie.

el tercer muestreo, M. americanum presentó tallas de aproximadamente $15 \mathrm{~cm}$ de longitud total, sin considerar los quelípedos. En la confluencia de los ríos Yuviaga y Copalita la diversidad de crustáceos cambió notablemente, de las 4 especies de langostinos sólo M. americanum y $M$. occidentale se capturaron en 2 muestreos, el primero y el tercero; M. digueti (Bouvier, 1895) y M. michoacanus se presentaron en el segundo y en el tercer muestreo. Los átyidos fueron más constantes; Atya sp. 1 apareció en los 3 muestreos, particularmente en el tercero, con el mayor número de organismos (juveniles); A. margaritacea se presentó en el primero y el tercero, con números similares (4 y 6, respectivamente) y $P$. glabra se registró en el segundo y el tercero, presentándose con mayor abundancia en el segundo muestreo. La presencia de cangrejos de río en un arroyo pequeño, manantial Piedras Negras, tributario del río Yuviaga, puede considerarse aparte de los grupos registrados en el sistema Yuviaga-Copalita, pues aunque se les buscó en ambos ríos, los resultados fueron negativos. La forma capturada fue Pseudothelphusa sp. 1.

En la porción baja se muestreó en el puente de la carretera HuatulcoSalina Cruz, donde la riqueza de especies y abundancia fue menor que en la porción baja de los otros ríos estudiados. Los átyidos fueron los mejor representados con Atya sp. 1 y P.glabra; en las capturas, los palemónidos aparecieron con frecuencia aunque con escaso número de ejemplares. Destacó la presencia de juveniles de Macrobrachium sp., en especial durante la cuarta salida, en la cual se observaron miles de estos organismos en la vegetación ribereña.

En el río Zimatán, en la porción alta de la cuenca, se presentó una dominancia de los átyidos y pseudotelfúsidos, cuyas especies se presentaron en los 4 muestreos. Por su abundancia destacó $A$. margaritacea; la composición de sexos fue equilibrada y durante la tercera y cuarta salidas se registraron hembras ovígeras y juveniles. Pseudothelphusa sp. 1 se colectó en todos los muestreos. Los palemónidos fueron escasos y únicamente se capturaron en la segunda salida, Macrobrachium occidentale con 3 ejemplares, $M$. michoacanus con 2 y M. americanum con 1; los juveniles aparecieron en el tercer muestreo y en el cuarto.

En el puente Petatengo, cuenca media, Potimirim glabra fue la especie dominante tanto en frecuencia de aparición como en número de organismos, en total se capturaron 254 organismos (124 $\delta^{\top}: 130$ P) incluyendo 24 hembras ovígeras. Otras especies como Atya sp. 1, M. michoacanus y Pseudothelphusa sp. 2, también se registraron en los 4 muestreos. En la porción baja, en el puente de la carretera Huatulco-Salina Cruz, la riqueza de especies fue alta, también se presentaron todas las formas 


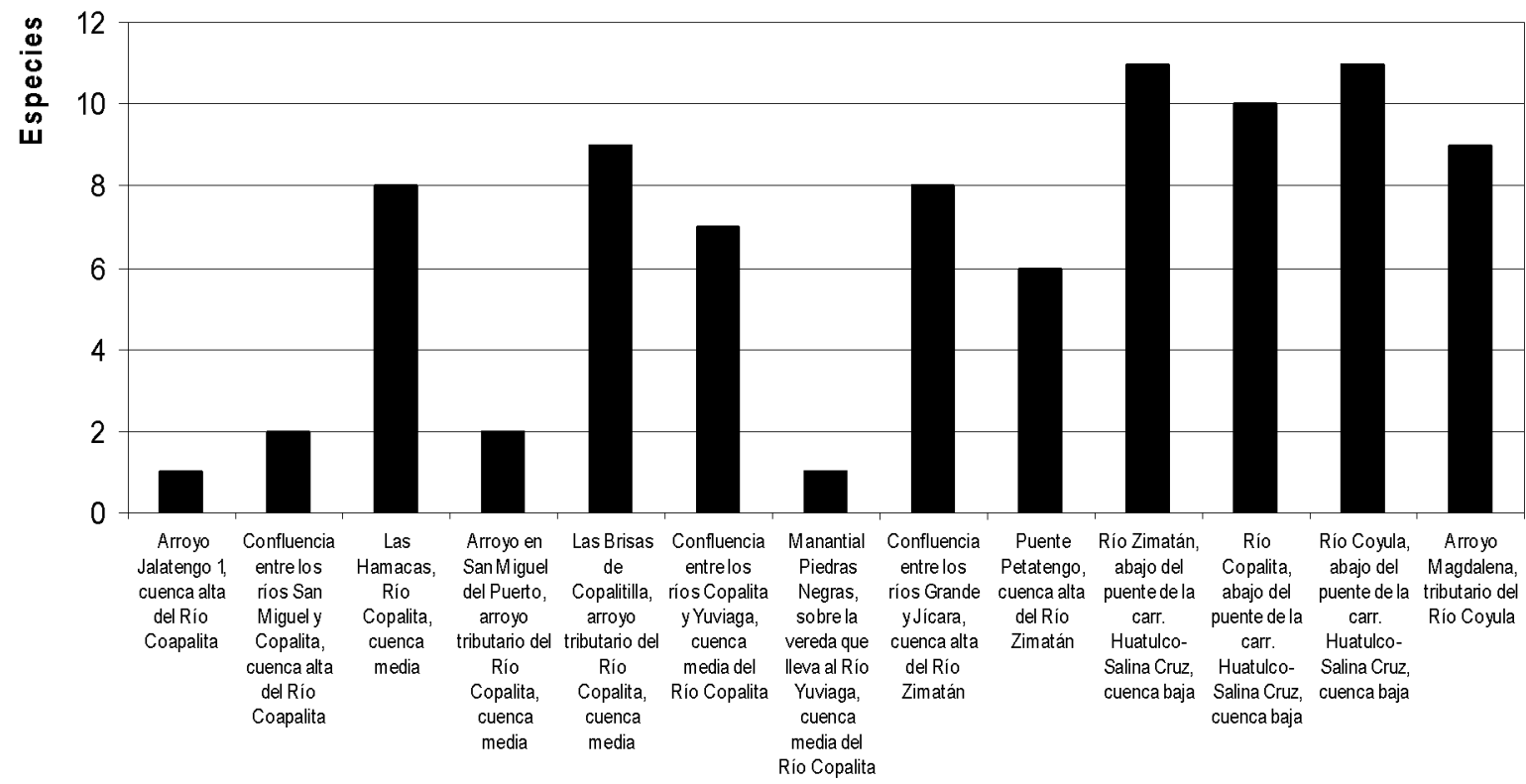

Localidades

Figura 4. Número de especies por localidad.

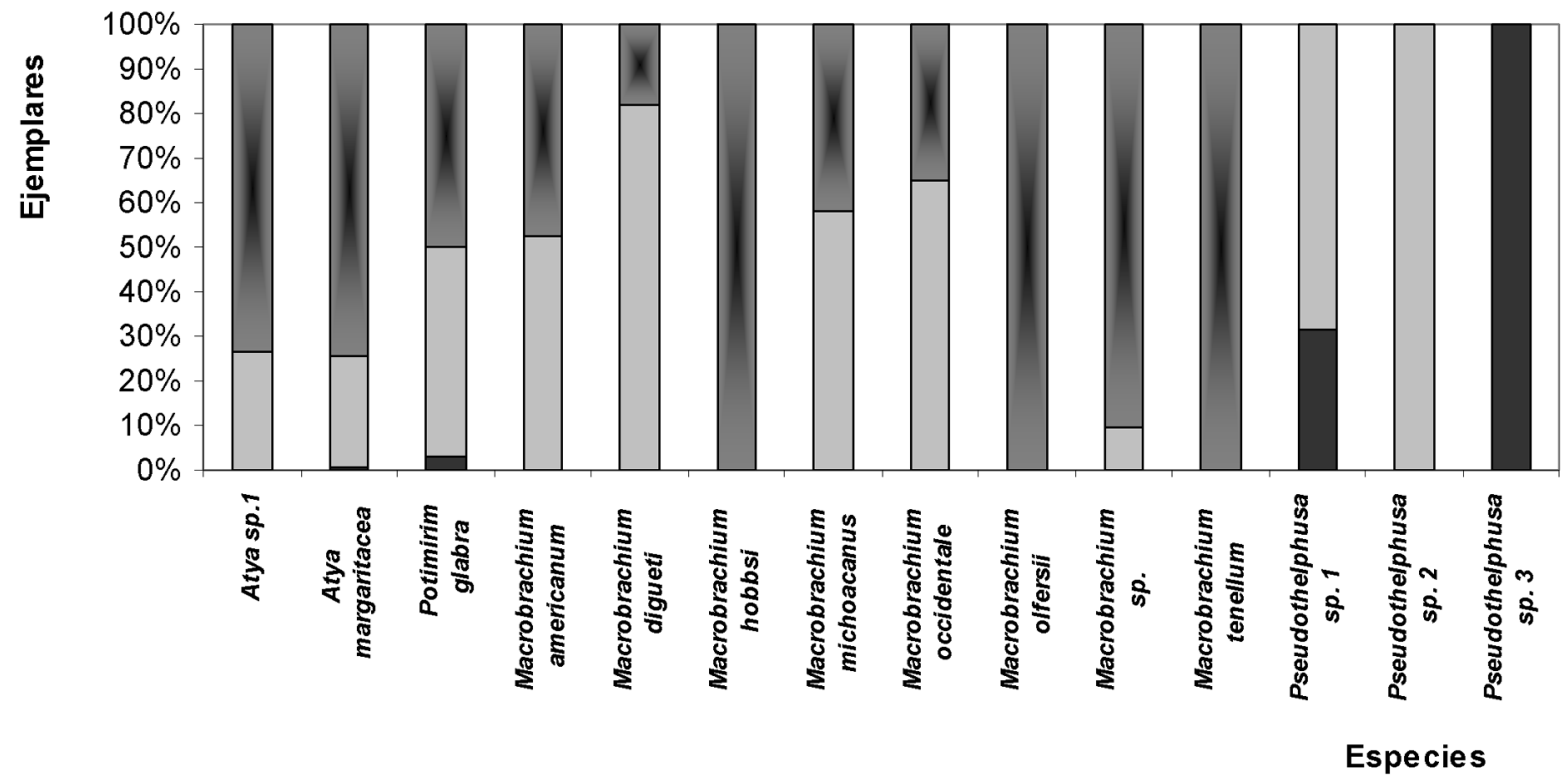

$\square$ ALTA $\square$ MEDIA $\square$ BAJA

Figura 5. Proporción de los ejemplares por especie obtenidos en las 3 zonas altitudinales de las cuencas estudiadas.

de átyidos y palemónidos. Sobresalió $M$. tenellum como la especie más abundante, y los juveniles de langostino se obtuvieron en los 4 muestreos en grandes números.

En el río Coyula en la localidad de arroyo Magdalena, cuenca media, dominaron los átyidos, las 3 especies se registraron en los 3 muestreos. La aparición de Atya sp. 1 fue más regular en número de ejemplares; en contraste, Potimirim glabra mostró un incremento evidente en el tercer muestreo; la proporción de sexos fue equilibrada (29 万̃: 31 +). Entre los palemónidos M. michoacanus se 
presentó en las 3 visitas y M. occidentale en 2. Por su parte, Pseudothelphusa sp. 2 se registró con un ejemplar en cada muestreo. En la porción baja, en la localidad del puente de la carretera Huatulco-Salina Cruz, se observó mayor contaminación por la influencia del poblado de Santa María Huatulco. La riqueza de especies fue alta, se encontraron todas las formas de átyidos y palemónidos citadas en el presente estudio. Atya margaritacea y Macrobrachium americanum se registraron en los 4 muestreos; Atya sp. 1, Potimirim glabra, M. hobbsi Nates-Rodríguez y Villalobos-Hiriart, 1990, M. occidentale y M. tenellum aparecieron en tres; $M$. digueti y $M$. olfersii (Wiegmann, 1836), en 1 sólo . Por su abundancia sobresalieron Atya sp. 1 y $P$. glabra.

Análisis de las especies por su distribución altitudinal. Se registraron tanto especies dulceacuícolas estrictas como anfídromas que durante la época de reproducción realizan migraciones hacia la porción baja de los ríos, para desovar cerca de la desembocadura al mar (McDowall, 2007) (Fig. 5). Entre las dulceacuícolas estrictas, Pseudothelphusa sp. 1 se capturó en la porción media y en la alta de las 3 cuencas estudiadas, siendo la única especie que se encontró en la altitud del arroyo Jalatengo (1 420 m). Pseudothelphusa sp. 2, en la porción media de los ríos Zimatán y Coyula, en las localidades de Petatengo y en el arroyo Magdalena, y Pseudothelphusa sp. 3, en la porción alta del río Copalita en la localidad de la confluencia con el río San Miguel (Fig. 5).

En cuanto a las formas anfídromas, Atya sp. 1 se registró en la porción media de las cuencas $(27 \%)$ principalmente con adultos, y en la porción baja (73\%) con predominancia de juveniles. Atya. margaritacea tuvo una distribución altitudinal semejante a la especie anterior, pero se capturó un ejemplar en la confluencia de los ríos San Miguel y Copalita (885 m), localidad considerada de la porción alta del río Copalita.

El 73\% de los ejemplares se capturó en las estaciones de la planicie costera, encontrándose juveniles, adultos y hembras ovígeras. Potimirim glabra también mostró un amplio intervalo de distribución altitudinal, desde las localidades ubicadas en la porción alta de las 3 cuencas hasta la planicie costera; junto con A. margaritacea se les puede considerar como las especies más comunes del estudio. Macrobrachium americanum se distribuyó en las porciones media y baja de las cuencas, con una proporción de ejemplares casi similar en ambas (50\%). Macrobrachium digueti presentó mayor abundancia en la porción media $(80 \%)$ y menor $(20 \%)$ en la porción baja. El mayor número de ejemplares de esta especie apareció durante la época de lluvias. Macrobrachium hobbsi fue una especie rara que se registró en las estaciones de la porción baja. Macrobrachium michoacanus se presentó en las porciones
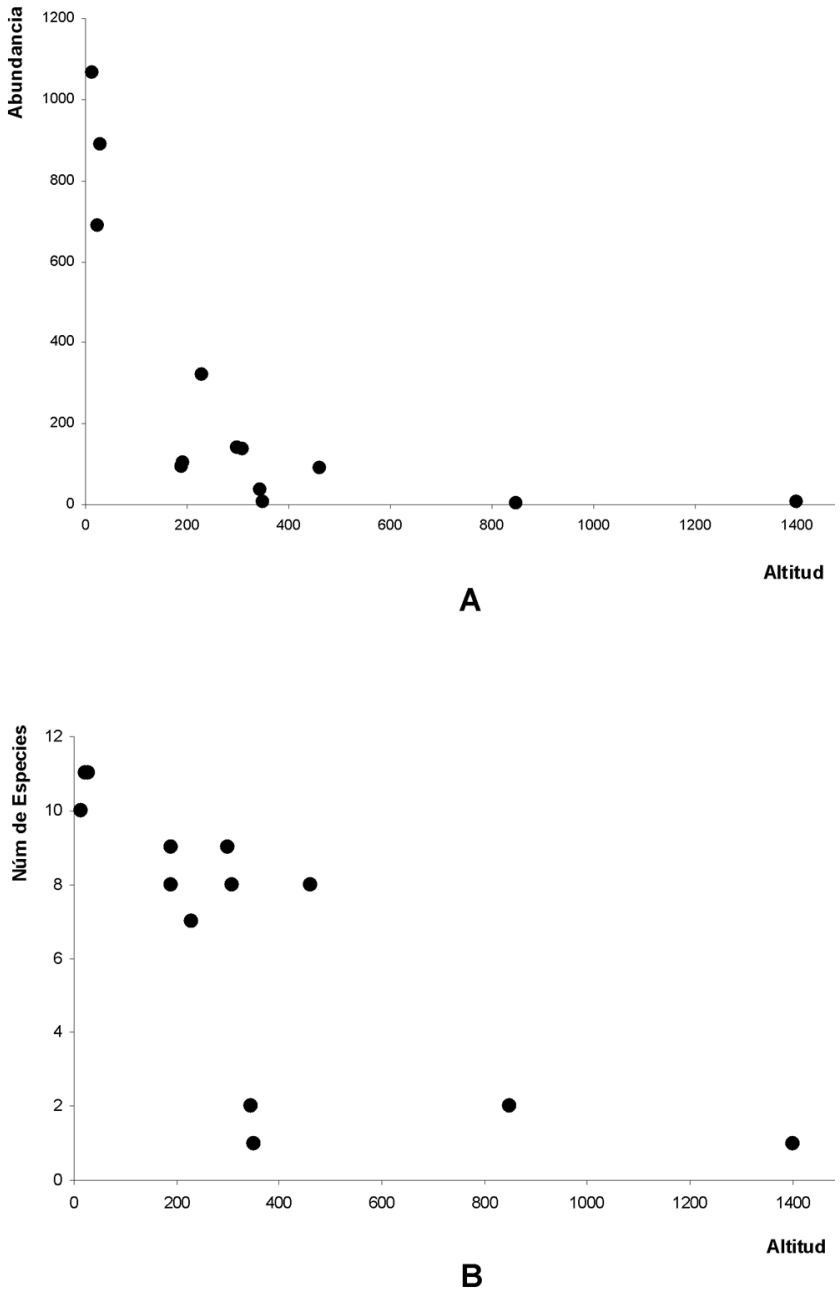

Figura 6. Influencia de la altitud sobre la composición de los decápodos colectados: A, abundancia vs. altitud; $\mathrm{B}$, riqueza de especies vs. altitud.

media (60\%) y baja (40\%) en una combinación de adultos, incluyendo hembras ovígeras y juveniles. Macrobrachium occidentale apareció con mayor frecuencia en la porción media (65\%) que en la porción baja (35\%). Macrobrachium olfersii tuvo una distribución restringida a la porción baja, su presencia fue irregular y escasa, sólo en el río Zimatán se encontró en los 4 muestreos. La presencia de juveniles de Macrobrachium se registró en la porción baja (90\%), el resto de los juveniles (10\%) se ubicó en la parte media. Macrobrachium tenellum se encontró solamente en la planicie costera.

Es claro que la abundancia total de las especies decrece de manera exponencial con la altitud (Fig. 6A). En las estaciones por debajo de $100 \mathrm{~m}$, las abundancias superan los 700 organismos, mientras que en las estaciones 

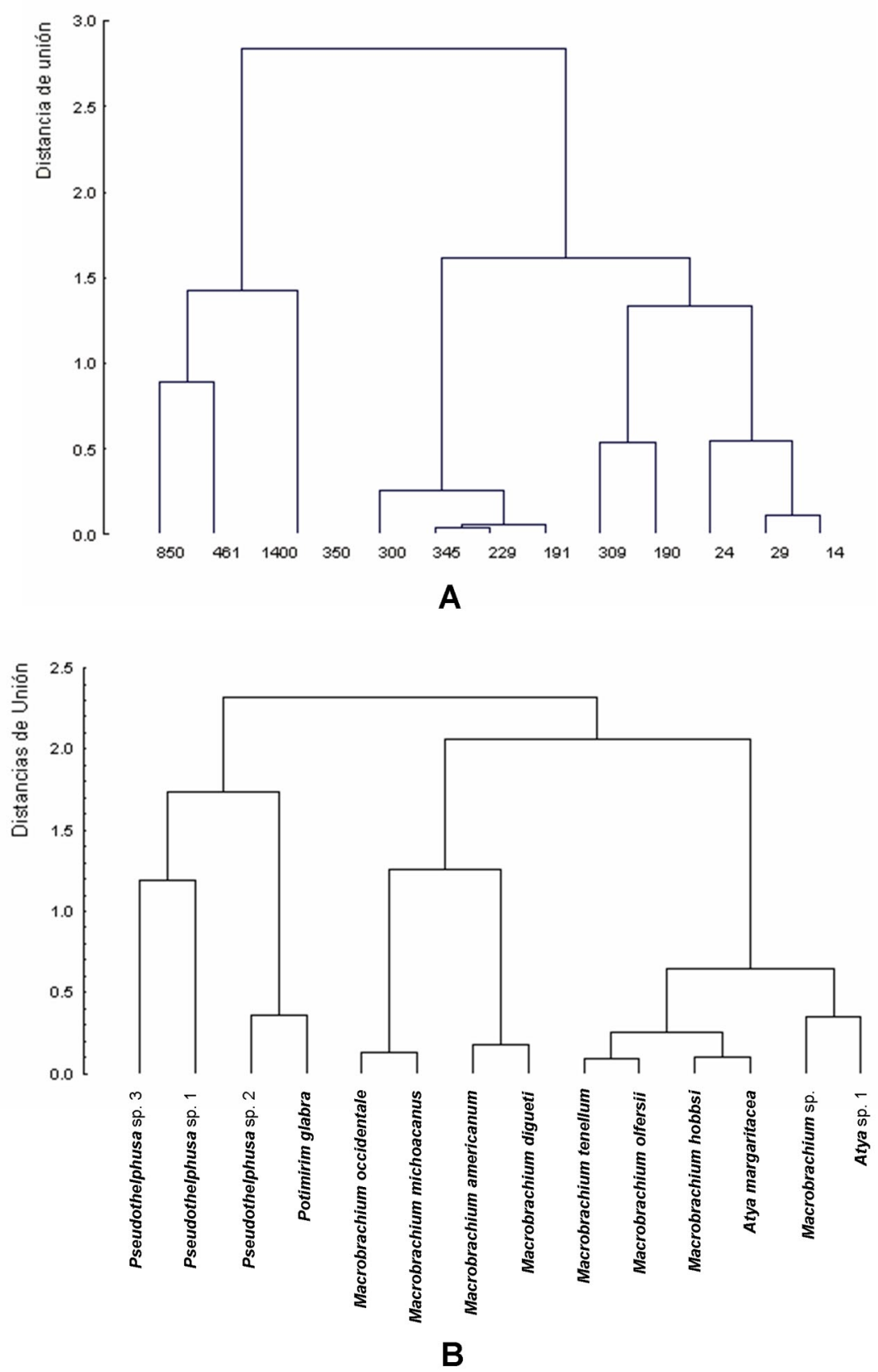

Figura 7. Análisis de agrupamiento realizado por el método de Ward utilizando las abundancias de cada especie por localidad: A, agrupamiento de localidades según su altitud; B, agrupamiento de especies de acuerdo con el intervalo altitudinal en el que se presentaron. Confluencia entre San Miguel y Copalita (850 m); arroyo Jalatengo (1 400 m); confluencia entre Grande y Jícara (461 m); manantial Piedras Negras (350 m); arroyo Magdalena (300 m); arroyo en San Miguel del Puerto (345 m); puente Petatengo (229 m); Las Hamacas (191 m); confluencia entre Yuviaga y Copalita (309 m); Las Brisas de Copalitilla (190 m); río Coyula parte baja (24 m); río Zimatán parte baja (29 m); río Copalita parte baja (14 m). con altitudes superiores a $200 \mathrm{~m}$, las abundancias fueron menores de 400 organismos.

Con respecto a la riqueza de especies se observa el mismo patrón, aunque aquí el decremento es más gradual (Fig. 6B). El máximo número de especies (11) se alcanza en las estaciones por debajo de los $100 \mathrm{~m}$ de altitud, decreciendo hasta llegar a una sola especie a los 1400 metros.

El análisis de agrupamiento de las localidades mostró la presencia de 3 grupos que coinciden con la idea de que las cuencas se pueden dividir en alta, media y baja. En la figura 7A se aprecia que las 3 localidades de mayor altitud se agrupan. Después hay otro agrupamiento de altitud media y otro más en altitudes bajas. El análisis por especie de acuerdo con el intervalo altitudinal (Fig. 7B) muestra que cada uno de los 3 grupos tiene, en efecto, especies características.Un caso especial en este estudio es $P$. glabra con un intervalo de distribución altitudinal muy amplio y que puede presentarse en diferentes grupos, dependiendo de la técnica de agrupamiento; aquí se presenta con el de especies que se distribuyen en la porción alta de las cuencas.

Análisis de las cuencas por la diversidad de su carcinofauna. La riqueza y diversidad de especies se incrementaron hacia la porción baja de las cuencas. Sin embargo, algunas localidades de la porción media de las 3 cuencas presentaron índices de diversidad casi tan altos como las localidades de la planicie costera, aunque en estas últimas el índice de equidad fue más bajo y el de dominancia más alto, lo que en parte refleja la mayor presencia de los organismos juveniles de Atya sp. 1, P. glabra y Macrobrachium sp.

En la cuenca del Copalita, resaltaron Las Hamacas y la confluencia de los ríos Yuviaga y Copalita, localidades que a pesar de tener una riqueza de especies similar (8), tuvieron un índice de diversidad diferente (2.71 y 2.857 


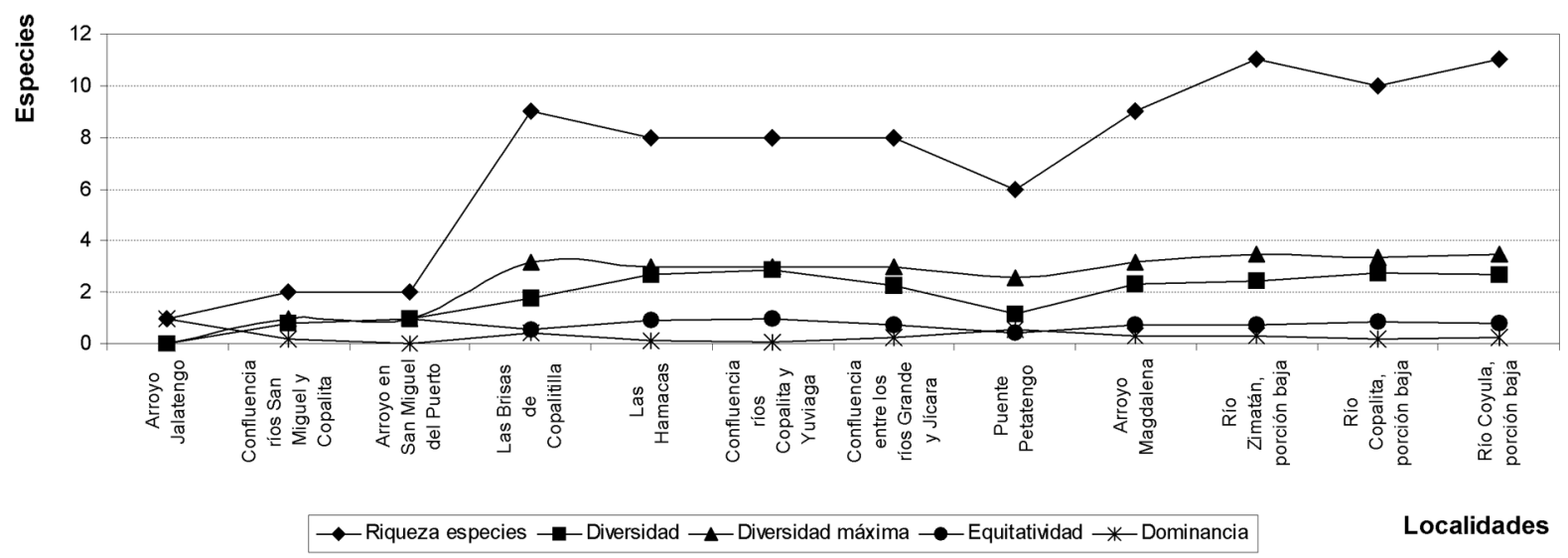

Figura 8. Representación gráfica de los valores obtenidos para los índices de diversidad, diversidad máxima, equitatividad y dominancia de acuerdo con el modelo de Shanon-Wiener, por localidad.

bits, respectivamente; Fig. 8), debido a las diferencias en los valores de equidad y dominancia que presentaron (0.903 y $0.952 ; 0.096$ y 0.047 , respectivamente).

En la cuenca del Zimatán destacó la localidad en la confluencia de los ríos Grande y Jícara con los valores más altos para los 3 índices. Puente Petatengo tuvo los valores más bajos, no sólo de su cuenca, sino del resto de las localidades ubicadas en la porción media.

En la cuenca del Coyula, la única estación de la porción media fue el arroyo Magdalena, la cual tuvo un índice de diversidad moderadamente alto, su equitatividad fue baja y la dominancia alta.

\section{Discusión}

De acuerdo con la literatura consultada y con los registros obtenidos de la CNCR, actualmente se conocen 36 especies de decápodos que se distribuyen en los cuerpos de agua epicontinentales de Oaxaca; pertenecen a las familias Alpheidae (1), Atyidae (7), Cambaridae (5), Palaemonidae (12), Pseudothelphusidae (9) y Trichodatylidae (2), lo que destaca al estado como el tercero en diversidad después de Chiapas y Veracruz. Entre otros factores, esta riqueza se debe a que en Oaxaca se distribuyen especies de ambas vertientes del país y varias de ambientes cavernícolas. Aunque en este estudio sólo se encontró el $27 \%$ de esa diversidad, se debe mencionar que 4 de las 13 especies reconocidas tienen la posibilidad de convertirse en especies nuevas o en extensiones de distribución geográfica de crustáceos citados para estados vecinos, como Guerrero y Veracruz. La presencia de posibles nuevas especies es común en ciertas regiones de México que han sido pobremente muestreadas o en grupos de crustáceos poco estudiados, como los cangrejos de agua dulce de la familia Pseudothelphusidae, que sólo en las últimas 2 décadas han sido revisados con intensidad (Villalobos-Hiriart, 2005; Villalobos y Álvarez, 2008).

La riqueza de especies obtenida concuerda con los ambientes fluviales muestreados, ya que son localidades de la vertiente occidental oaxaqueña donde son comunes las familias Atyidae, Palaemonidae y Pseudothelphusidae. Los representantes de las otras familias (Alpheidae, Cambaridae y Trichodactylidae) se distribuyen en ambientes muy particulares de la franja colindante con el estado de Veracruz (Hobbs, 1973a, b; Rodríguez, 1992; Rojas-Paredes, 1998; Mejía-Ortíz, 2005).

Los valores de abundancia y riqueza de especies decrecieron conforme aumentó la altitud. Este patrón ya fue descrito para varias especies de Macrobrachium en Veracruz (Mejía-Ortiz el at., 2001; Mejía-Ortiz y Álvarez, 2010). Los resultados de este estudio confirman el patrón con un conjunto de especies diferentes.

Los átyidos y palemónidos son los crustáceos más frecuentes en las porciones media y baja de las vertientes occidental y oriental del país. Varias especies de estas familias, como A. margaritacea y $M$. tenellum, debido a su talla y abundancia, son de importancia comercial para la localidad y para el estado (Rodríguez de la Cruz, 1965; Villalobos-Figueroa et al., 1982; Martínez-Mayén et al., 2000; Lorán-Nuñez et al., 2009).

La pesquería de crustáceos en las cuencas estudiadas es 
totalmente artesanal. Los pobladores de la zona aprovechan el incremento del cauce de los ríos durante la época de lluvias para llevar a cabo la pesquería de langostinos de grandes tallas como M. americanum o de tallas moderadas como $M$. digueti y $M$. occidentale, e incluso de átyidos como A. margaritacea. Las hembras ovígeras de átyidos y palemónidos se valen de las grandes avenidas para realizar sus movimientos de las cabeceras hacia la porción baja de los ríos con el propósito de liberar sus larvas en los ambientes estuarinos donde encuentran las condiciones favorables para iniciar su desarrollo (Jalihal et al., 1993; Rome et al., 2009). Mejía-Ortiz y Álvarez (2010) describen un proceso similar de alta conectividad entre diferentes zonas del río Huitzilapan, Veracruz, debido a un mayor volumen de agua durante la época de lluvias, en contraste con una diferenciación de varias zonas del río durante la época de secas. Este patrón coincide con la redistribución de tallas de diversas especies a lo largo de los ríos.

La porción baja de los ríos estudiados tiene gran importancia para los crustáceos reconocidos, ya que son áreas de crianza y protección para las larvas. En todas estas localidades se encontraron hembras ovígeras y juveniles de la mayoría de las especies.

Las desembocaduras de los ríos y los tramos de estos cuerpos fluviales enmarcados en la planicie costera desempeñan un papel fundamental en el repoblamiento de las porciones media y alta de los ríos, tanto de crustáceos como de peces con conductas anfídromas (McDowall, 2007; Bauer y Delahoussaye, 2008). Paradójicamente, suelen ser las zonas más codiciadas para los asentamientos humanos, construcción de desarrollos turísticos, desagüe de desechos urbanos o para la apertura de campos agrícolas.

Si bien, la conducta migratoria es el patrón general de las especies de átyidos y palemónidos capturados, en las localidades de Las Brisas de Copalitilla, Las Hamacas y en la confluencia de los ríos Copalita y Yuviaga, así como en la de los ríos Grande y Jícara, que se encuentran a una distancia considerable de la desembocadura de los ríos Copalita y Zimatán, fue evidente la presencia de juveniles de ambas familias, a pesar de que sería imposible para estos juveniles realizar la migración de la zona estuarina a la porción media de las cuencas citadas sin sufrir cambios en su tamaño y estado de madurez. Encontrar fases juveniles propias de las desembocaduras de los ríos en las partes altas es un fenómeno que se presenta en varios ríos de México, donde es posible que otro tipo de sales $\left(\mathrm{CaCO}_{3}\right.$, $\mathrm{MgCO}_{3}$ ) disueltas en el agua se conviertan en sustituto de las sales marinas $(\mathrm{NaCl})$. Estas condiciones se presentan en arroyos o ríos que por la composición del sustrato de sus cuencas y la disolución de elementos cársticos le dan al agua cierta dureza que permite que las especies completen su ciclo biológico sin necesidad de realizar migraciones hacia la porción baja de la cuenca fluvial. Se trata de aguas carbonatadas cálcicas que dado su paso a través de rocas calizas presentan baja salinidad, toda vez que drenan rocas graníticas y gneis. Esta suposición está apoyada por los valores de salinidad registrados en localidades de las porciones alta y media de las cuencas estudiadas (Las Brisas de Copalitilla, 0.13-0.16 ups; Las Hamacas, 0.10-0.12 ups; confluencia de los ríos Copilita y Yuviaga, 0.11-0.15 ups; confluencia de los ríos Grande y Jícara, 0.90.15 ups), las cuales mostraron valores muy similares a los obtenidos en las localidades de la porción baja (Puente Zimatán, 0.10-0.16 ups; Puente Copalita, 0.10-0.12 ups; Puente Coyula, 0.10-0.14 ups) (De La Lanza, 2009).

\section{Literatura citada}

Álvarez, F. y J. L. Villalobos. 1990. Pseudothelphusa galloi, new species of freshwater crab (Brachyura: Pseudothelphusidae) from southwestern Mexico. Proceedings of the Biological Society of Washington 103:103-105.

Álvarez, F. y J. L. Villalobos. 1991. Two new freshwater crabs from Mexico, Odontothelphusa toninae and Stygothelphusa lopezformenti (Crustacea, Brachyura, Pseudothephusidae). Proceedings of the Biological Society of Washington 104:288-294.

Bauer, R. T. y J. Delahoussaye. 2008. Life history migrations of the amphidromous river shrimp Macrobrachium ohione from a continental large river system. Journal of Crustacean Biology 28:622-632.

De La Lanza, G. 2009. Informe final: propuesta de indicadores de la salud de la cuenca para el monitoreo biológico del sistema hidrológico Copalita-Zimatán-Huatulco, Oax. World Wildlife Fund-México.

González-Mora, I. D., G. de la Lanza-Espino y R. SánchezNavarro. 2009. Memoria del Taller: propuesta de caudal ecológico en la cuenca Copalita-Zimatán-Huatulco, 2009. Manejo del agua en cuencas hidrográficas: desarrollo de nuevos modelos en México. WWF / Fundación Gonzálo Río Arronte, I.A.P., México, D.F. 19 p.

Gray, J. 1981. The ecology of marine sediments (An introduction to the structure and fuction of benthic communities). Cambridge University Press, New York. 185 p.

Hernández, L., G. Murugan, G. Ruis-Campos y A. MaedaMartínez. 2007. Freshwater shrimp of the genus Macrobrachium (Decapoda: Palaemonidae) from the Baja California Peninsula, Mexico. Journal of Crustacean Biology 27:351-369.

Hobbs, H. H., Jr. 1973a. Three new troglobitc decapods crustaceans from Oaxaca, Mexico. Association for Mexican Cave Studies Bullentin 5:25-38.

Hobbs, H. H., Jr. 1973b. Two new troglobitc shrimps (Decapoda: Alpheidae and Palaemonidae) from Oaxaca, Mexico. Association for Mexican Cave Studies Bulletin 5:73-80.

Hobbs, H. H., Jr. 1989. An illustrated checklist of the American crayfishes (Decapoda: Astacidae, Cambaridae, and 
Parastacidae). Smithsonian Contributions to Zoology 480:1236.

Hobbs, H. H., Jr. y C. W. Hart, Jr. 1982. The shrimp genus Atya (Decapoda: Atyidae). Smithsonian Contributions to Zoology 364:1-143.

Holthuis, L. B. 1952. A general revision of the Palaemonidae (Crustacea Decapoda Natantia) of Americas, II: The subfamily Palaemoninae. Allan Hancock Foundation Occasional Papers 12:1-396.

Holthuis, L. B. 1977. Cave shrimp (Crustacea, Decapoda, Natantia) from Mexico. Accademia Nazionale dei Lincei 171:173-195.

INEGI, 1996. Carta Geológico-Minera Puerto Escondido D143, Oaxaca, Esc. 1:250,000. http://www.sgm.gob.mx/; última consulta: 06.VI.2009.

Jalihal, D. K., K. N. Sankolli y S. Shenoy. 1993. Evolution of larval development patterns and the process of freshwaterization in the prawn genus Macrobrachium Bate, 1868 (Decapoda: Palaemonidae). Crustaceana 65:365-376.

Lorán-Nuñez, R. M., A. J. Valdéz-Guzmán y F. R. MartínezIsunza. 2009. Estudio biológico-pesquero del "burro" Atya scabra en el río de los Pescados y río Actopan, Veracruz, México. Ciencia Pesquera 17:5-16.

Martínez-Mayén, M., R. Román-Contreras, A. Rocha-Ramírez y S. Cházaro-Olvera. 2000. Relative growth of Atya margaritacea A. Milne-Edwards, 1864 (Decapoda, Atyidae) from the southern Pacific coast of Mexico. Crustaceana 73:525-534.

McDowall, R. M. 2007. On amphidromy, a distinct form of diadromy in aquatic organisms. Fish and Fisheries 8:1-13.

Mejía-Ortiz, L. M. 2005. Adaptations to cave life in decapods from Oaxaca. Association for Mexican Cave Studies Bulletin 15:1-169.

Mejía-Ortiz, L. M. y F. Álvarez. 2010. Seasonal patterns in the distribution of three species of freshwater shrimp, Macrobrachium spp., along an altitudinal river gradient. Crustaceana 83:385-397.

Mejía-Ortiz, L. M., R. G. Hartnoll y J. A. Viccon-Pale. 2003. A new stygotic crayfish from México, Procambarus cavernicola (Decapoda: Cambaridae), with a review of cave-dwelling crayfishes in Mexico. Journal of Crustacean Biology 23:391-401.

Mejía, L. M., F. Álvarez y R. G. Hartnoll. 2003. A new species of freshwater prawn, Macrobrachium totonacum (Decapoda, Palaemonidae), with abbreviated development from Mexico. Crustaceana 76:77-86.

Mejía-Ortiz, L. M., F. Álvarez, R. Román y J. A. Viccon-Pale. 2001. Fecundity and distribution of freshwater prawns of the genus Macrobrachium in the Huitzilapan River, Veracruz, Mexico. Crustaceana 74:69-77.

Nates-Rodríguez, J. C. y J. L. Villalobos-Hiriart. 1990. Dos especies nuevas de camarones de agua dulce del género Macrobrachium Bate, (Crustacea, Decapoda, Palaemonidae), de la vertiente occidental de México. Anales del Instituto de Biología, Serie Zoología 61:1-11.

Rathbun, M. J. 1898. A contribution to a knowledge of the freshwater crabs of America. The Pseudothelphusinae. Proceedings of the United States National Museum 21:507-537.
Rathbun, M. J. 1933. A new species of Pseudothelphusa from Mexico. Journal of the Washington Academy of Science 23:360.

Rodríguez, G. 1982. Les crabes d'eau douce d'Amerique. Famille des Pseudothelphusidae. Faune Tropicale 22:1-223.

Rodríguez, G. 1992. The freshwater crabs of America. Faune Tropicale 31:1-189.

Rodríguez, G. y A. E. Smalley. 1969. Los cangrejos de agua dulce de México de la familia Pseudothelphusidae (Crustacea, Brachyura). Anales del Instituto de Biología, Serie Zoología 40:69-112.

Rodríguez de la Cruz, M. C. 1965. Contribución al conocimiento de los Palemónidos de México II. Palemónidos del Atlántico y vertiente oriental de México con descripción de dos especies nuevas. Anales del Instituto Nacional de Investigaciones Biológico Pesqueras 1:70-112.

Rojas-Paredes, Y. R. 1998. Revisión taxonómica de 8 especies del género Procambarus (Crustacea: Decapoda: Cambaridae) del centro de Veracruz, México. Tesis, Facultad de Ciencias, Universidad Nacional Autónoma de México, México, D.F. $158 \mathrm{p}$.

Rome, N. E., S. L. Conner y R. T. Bauer. 2009. Delivery of hatching larvae to estuaries by an amphidromous river shrimp: tests of hypothesis based on larval moulting and distribution. Freshwater Biology 54:1924-1932.

Salas-Morales, S. H., A. Saynes-Vásquez y L. Schibli. 2003. Flora de la costa de Oaxaca, México: Lista florística de la región de Zimatán. Boletín de la Sociedad Botánica de México 72:21-58.

Tamayo, J. L. 1949. Geografía general de México. Geografía física, tomo II. Talleres Gráficos de la Nación, México, D.F. $580 \mathrm{p}$.

Villalobos, A. 1948. Estudios de los cambarinos mexicanos, VII. Descripción de una nueva especie del género Procambarus. Procambarus acanthophorus n. sp. Anales del Instituto de Biología, Universidad Nacional Autónoma de México 19:175-182.

Villalobos, A. 1954. Estudios de los cambarinos mexicanos, XII, parte 1. Revisión de las especies afines a Procambarus mexicanus (Erichson), con descripción de nuevas formas. Anales del Instituto de Biología, Universidad Nacional Autónoma de México 25:299-379.

Villalobos, A. 1955. Cambarinos de la fauna mexicana (Crustacea, Decapoda). Tesis doctorado, Facultad de Ciencias, Universidad Nacional Autónoma de México, 290 p.

Villalobos, A. 1956. Contribución al conocimiento de los Atyidae de México, I: Una nueva especie de Atya de la vertiente del Pacífico del estado de Michoacán. Anales del Instituto de Biología, Universidad Nacional Autónoma de México 26:459-475.

Villalobos, A. 1960. Contribución al conocimiento de los Atyidae de México. II (Crustacea, Decapoda). Estudio de algunas especies del género Potimirim (= Ortmannia), con descripción de una especie nueva en Brasil. Anales del Instituto de Biología, Universidad Nacional Autónoma de México 30:269-330.

Villalobos, A. 1967. Estudio de los palaemónidos de México I. Macrobrachium acanthochirus n. sp. del sudoeste de 
México. Anales del Instituto de Biología, Universidad Nacional Autónoma de México 25:167-174.

Villalobos, A. 1969. Problemas de especiación en América de un grupo de Palaemonidae del género Macrobrachium. Actas de la Conferencia Científica Mundial sobre Biología y Cultivo de Camarones y Gambas. FAO Fisheries Reports 3:1055-1066

Villalobos, A. 1982. Decapoda. In Aquatic biota of Mexico, Central America and the West Indies, S. H. Hulbert y A. Villalobos (eds.). San Diego State University, California, pp. 215-239.

Villalobos-Figueroa, A., M. E. Zamora-Sánchez, J. J. CorreaLópez, J.L.Espinosa-Aranda y M. L. Nieto-Applebaum.1982. Evaluación de la disponibilidad de postlarvas de Macrobrachium tenellum (Smith) y determinación de sus posibilidades de semicultivo en las microrregiones PIDER, Costa Grande y Atoyac del estado de Guerrero. Informe final. Delegación Estatal de Pesca del estado de Guerrero, Chilpancingo, Gro. Inédito.

Villalobos-Hiriart, J. L. 2005. Sistemática de los cangrejos de agua dulce de México, Tribu Pseudothelphusini Ortmann, 1897
(Crustacea: Decapoda: Brachyura: Pseudothelphusidae). Análisis filogenético, biogeográfico y descripción de especies nuevas. Tesis doctorado, Facultad de Ciencias, Universidad Nacional Autónoma de México. 394 p.

Villalobos, J. L. y F. Álvarez. 2003. Two new species of freshwater crabs of the genus Tehuana (Brachyura: Pseudothelphusidae) from southern Mexico. Journal of Crustacean Biology 23:223-229.

Villalobos, J. L. y F. Álvarez. 2008. Los cangrejos de agua dulce de la familia Pseudothelphusidae (Decapoda: Brachyura: Eubrachyura) de México. Con un apéndice de las especies citadas para América hasta el 2006. In Crustáceos de México: estado actual de su conocimiento, F. Álvarez y G. Rodríguez (eds.) PROMEP-UANL, Secretaría de Educación Pública / Dirección General de Publicaciones, Universidad Autónoma de Nuevo León, Monterrey. p. 239-299.

Villalobos-Hiriart, J. L., A. Cantú Díaz-Barriga y E. LiraFernández. 1993. Los crustáceos dulceacuícolas de México. In Diversidad biológica en México, R. Gío-Argáez y E. López-Ochoterena (eds.). Revista de la Sociedad Mexicana de Historia Natural, volumen especial 64:267-290. 